\title{
A retrospective comparison between transrectal and transperineal prostate biopsy in the detection of prostate cancer
}

\author{
Carmelo Agostino Di Franco ${ }^{1}$, Hussein Jallous ${ }^{1}$, Daniele Porru ${ }^{1}$, Giovanni Luca Giliberto ${ }^{1}$, \\ Tiziano Cebrelli ${ }^{1}$, Carmine Tinelli ${ }^{2}$, Bruno Rovereto ${ }^{1}$ \\ ${ }^{1}$ Urology Department, IRCCS Policlinico S. Matteo, Pavia, Italy; \\ 2 Biometrics and Medical Statistics Service, IRCCS Policlinico S. Matteo, Pavia, Italy.
}

\begin{abstract}
Summary Background: The aim of this study was to analyze the differences between TRUSguided transrectal prostate biopsy (TR) and transperineal prostate biopsy $(T P)$ in the diagnosis of prostate cancer. The two biopsy methods were evaluated in terms of diagnostic sensitivity and of early and late complications.

Methods: This retrospective study was realized through the review of clinical records of 219 men that received a prostate biopsy between 2004 and 2014. The biopsy was performed because of elevated prostate-specific antigen (PSA), abnormal digital rectal examination findings (DRE), abnormal transrectal ultrasound (TRUS) findings and symptoms due to prostate diseases. The cohort study was subdivided in two groups: 108 patients received a transrectal biopsy between 2004 and 2006 and 111 received a transperineal biopsy between 2007 and 2014. In both groups, first biopsy was performed with 12 cores scheme whereas second or third biopsy were performed with 18 cores scheme; in this study we excluded patients who underwent to biopsies with different number cores to reduce the bias. Both groups were evaluated on the basis of age, total PSA, PSA ratio (F/T), DRE/TRUS findings, presencelabsence of low urinary tracts symptoms (LUTS), presencelabsence of benign prostatic hyperplasia (BPH), histologic findings of biopsy cores and immediate/postoperative complications. Then, it was evaluated the overall cancer detection rate and the stratified cancer rate on the basis of the previous reported parameters. Finally, we analyzed the early and late complication rate in both groups. U Mann-Whitney test was used to evaluate the quantitative variables and $\chi^{2}$-test or Fisher exact test for qualitative variables. $p<0.05$ was considered statistically significant.
\end{abstract}

Results: 66 cancers were detected in 219 patients of the study; 29 cancers were detected in the TP group and 37 in the TR group. There were no statistically significant differences in the overall cancer rate detected in both groups (26.13\% e 34.26\% respectively; $p=0.190$ ). However, TP biopsy detected more cancers at first biopsy than TR biopsy (89.7\% vs $78.4 \%$ respectively; $p=0.021$ ). Moreover, TP biopsy detected more cancers in those patients with low cancer suspect (PSA $<4$ $n g / m l, F / T>15 \%$, negative TRUS), instead TR biopsy had more sensitivity in detecting cancer in those patients with high cancer suspect (PSA $>10 \mathrm{ng} / \mathrm{ml}, \mathrm{F} / \mathrm{T}<15 \%$, TRUS with abnormal lesions). The presence of BPH did not influence sensitivity in both cases. There were no significant differences in the early complication rate whereas a statistically significant difference was observed in the late complication rate (4\% vs $11 \%$ in TP and TR biopsy, respectively; $p=0.019$ ).
Conclusions: No statistically significant differences in sensitivity were observed between TP and TR biopsy, but TP biopsy detected more cancers at first time biopsy. Complications rate was lower in the TP group. Therefore, we conclude that the Urologist has the final choice in deciding the most appropriate biopsy technique, considering sensitivity and complications.

KEY WORDS: Urology; Oncology; Prostate; Biopsy; PSA; Prostatic cancer; Transperineal prostate biopsy; Transrectal prostate biopsy; Rectal exploration.

Submitted 1 February 2017; Accepted 28 February 2017

\section{INTRODUCTION}

The systematic sextant prostate biopsy, introduced in 1989 by Hodge et al. (1), has been for long time the gold standard for the diagnosis of prostate cancer. More recent studies have suggested that standard sextant biopsy may underestimate the incidence of cancer, with reported false negative rates of up to $31 \%$ (2). To improve the cancer detection rate it was included increasing the number of specimen cores and sampling extended fields of the prostate, in particular lateral prostate regions where usually cancers grow (3).

Most of American Urologists like to perform transrectal (TR) prostate biopsy, while in Asian and European countries some institutions prefer transperineal (TP) prostate biopsy. In our institute, the I.R.C.S.S. Policlinico S. Matteo of Pavia, TR biopsy was performed until 2006 when it was replaced by TP method. Today, prostate biopsy has been greatly improved with the introduction of prostatic magnetic resonance and the relative fusion-biopsy. However, magnetic resonance is an expensive exam and it is not yet available in all centers, especially in those minors.

We want to underline the need to have a diagnostic procedure to detect prostate cancer in the best way, by using traditional prostate biopsy. In this work we analyze, retrospectively, the differences in term of sensitivity and complications rate between transrectal and transperineal biopsies in prostate cancer diagnosis. 


\section{MATERIALS AND METHOdS}

We evaluated a cohort of 219 men, randomly chosen among the patients that received a prostate biopsy between 2004 and 2014. In our department transrectal prostate biopsy was performed until 2006 when it was replaced by transperineal prostate biopsy. The cohort was subdivided in two groups on the basis of the used technique: 111 patients in the transrectal biopsy group (TR) and 108 patients in the transperineal biopsy group (TP). In both groups, first biopsy was performed with 12 cores whereas second or third biopsy was performed with 18 cores; in this study we excluded patients who underwent to different number cores biopsies to reduce the bias. Patients charts were evaluated in a completely random way, without any criteria of inclusion/exclusion but the fact that patients received a prostate biopsy. The use of anticoagulant drugs was not evaluated because of reduced documentation about it. The prostatic volume was not evaluated because a very low patients number received a volume measurement before biopsy. Patients who received a TRUS before biopsy, were evaluated only as regards presence/absence of suspect nodules.

Whenever, in the study, we refer to BPH we use the clinic parameter or drugs utilization (such as alpha-lytic).

All the procedures were TRUS-guided. Table 1 shows the characteristics of patients assigned to transperineal and transrectal biopsy groups.

Prostate mapping was performed because of elevated prostate-specific antigen (PSA), abnormal digital rectal examination findings (DRE), abnormal transrectal ultrasound (TRUS) findings and symptoms due to prostate diseases. Each patient had his clinical and nursing charts, laboratory results and ultrasound and pathology reports. In particular, we focused on patients age, on DRE/TRUS findings (recorded as normal, benign or BPH and suspect for cancer), on total Prostatic Specific Antigen (tPSA) and PSA ratio, on presence/absence of lower urinary tract symptoms (LUTS) and on presence/absence of benign prostatic hypertrophy (BPH).

\section{Table 1.}

Patients' characteristics in two groups

(values $p<0.05$ are statistically significant).

\begin{tabular}{|l|c|c|c|}
\hline & TP (n. 111) & TR (n. 108) & P-value \\
\hline Median age (IQR) & 68 (IQR: 61-73) & 66 (IQR: 58.5-70.5) & 0.0192 \\
\hline Median PSA (IQR) & 6.9 (IQR: 4.94-10.03) & 7.8 (IQR: 5.24-12.3) & 0.1038 \\
\hline Median F/T (\%) (IQR) & 14.5 (IQR: 10.9-21) & $12.8($ IQR: 10-18.7) & 0.0703 \\
\hline Negative DRE* (\%) & $21(21.43 \%)$ & $38(36.19 \%)$ & 0.066 \\
Benign DRE* (\%) & $51(52.04 \%)$ & $43(40.95 \%)$ & \\
Suspect DRE* (\%) & $26(26.53 \%)$ & $24(22.86 \%)$ & \\
\hline Normal TRUS** (\%) & $4(10.53 \%)$ & $9(17.65 \%)$ & 0.165 \\
Benign TRUS** (\%) & $21(55.26 \%)$ & $18(35.29 \%)$ & \\
Suspect TRUS** (\%) & $13(34.21 \%)$ & $24(47.06 \%)$ & \\
\hline Present LUTS (\%) & $52(46.85 \%)$ & $45(42.06 \%)$ & 0.477 \\
Absent LUTS(\%) & $59(53.15 \%)$ & $62(57.94 \%)$ & \\
\hline Present BPH*** (\%) & $52(47.27 \%)$ & $42(40.38 \%)$ & 0.310 \\
Absent BPH*** (\%) & $58(52.73 \%)$ & $62(59.62 \%)$ & \\
\hline Abbreviations: TP: transperineal biopsy; TR: transrectal biopsy; PSA: prostate-specific antigen; \\
F/T: free PSA total PSA; DRE: digital rectal examination; TRUS: transrectal ultrasound; \\
LUTS: low urinary tract symptoms; BPH: benign prostatic hyperplasia. \\
*: avaliable in 203 pt; **: avaliable in 89 pt; ***: avaliable in 214 pt. \\
\hline
\end{tabular}

Complications occurred during or immediately after the biopsy were defined immediate whereas they were defined late if occurred during the post-operative observation. All the results were inserted in a Microsoft Excel database.

TR biopsies were performed using a preloaded 18-gauge biopsy needle inserted in a biopsy gun.

As regards pain control, TR biopsy was performed without anesthesia in 22 patients, with local anesthesia in 77 patients, with sedation in 9 patients. Local anesthesia was given through $2 \%$ lidocaine periprostatic injection using Chiba needle 22-gauge.

Overall, among the 108 men of TR group 132 biopsies were performed with 21 men receiving a second repeated biopsy and 3 men a third repeated biopsy. The indication to perform a further biopsy in the same patient was the constant PSA increase in spite of a first negative biopsy. All first biopsies were performed using a 12-core scheme biopsy; in second and third biopsies we used a 18-cores scheme. Finally, we checked the intra-glandular or rectal bleeding at the end of the procedure.

TP biopsies were performed using a preloaded 18-gauge biopsy needle inserted in a biopsy gun. All patients received local perineal and periprostatic anesthesia with $2 \%$ lidocaine.

Among the 111 patients of TP group, 125 biopsies were performed with 14 men receiving a second repeated biopsy. Also in this case, first biopsy had a 12-core scheme whereas in second and third biopsies we used an 18-cores scheme. At the end of both procedures, all patients were monitored in order to check the possibility of complications. They were discharged after the first asymptomatic urination.

In order to stratify patients we used total PSA, PSA ratio, DRE/TRUS findings (if available). Moreover, we evaluated the cancer detection rate (number of cancers/total patients number) in both procedures. Complications were divided in hematuria, dysuria/strangury, acute retention of urine, intra-glandular bleeding, rectal bleeding, vasovagal syncope, severe headache, cardiovascular complications (hypertensive crisis, arrhythmias, extra systoles).

The quantitative data were summarized with median and interquartile range because they are not normally distributed. On the other hand, qualitative variables were described as counts and percentages. Comparisons of quantitative variables of the two groups were realized by U Mann-Whitney test, whereas we used $\chi^{2}$-test or Fisher exact test to analyze the qualitative variables. All tests are two tailed and the significance level was 5\% $(\mathrm{p}<0.05)$. All analysis were performed using STATA software (ver.9.Stata Corporation, College Station, 2008, Texas, USA).

\section{Results}

Among 219 patients, 66 (30\%) received a prostate cancer diagnosis. In particular transperineal biopsy (TP) detected 29 cancers $(26.13 \%)$ in 111 patients, whereas 
Table 2.

Number of revealed cancers/overall number of revealed cancers by number of biopsies.

\begin{tabular}{|l|c|c|c|}
\hline & TP & TR & Risultati TP vs TR (\%) \\
\hline $1^{\circ}$ Biopsy & $26 / 29(89.7)$ & $29 / 37(78.4)$ & 89.7 vs 78.4 \\
\hline $2^{\circ}$ Biopsy & $3 / 29(10.3)$ & $6 / 37(16.2)$ & 10.3 vs 16.2 \\
\hline $3^{\circ}$ Biopsy & $0(0)$ & $2 / 37(5.4)$ & 0 vs 5.4 \\
\hline
\end{tabular}

transrectal biopsy (TR) detected 37 cancers (34.26\%) in 108 men; however there were no statically significant differences $(p=0.190)$ between two techniques. There was no evidence of PIN/ASAP in the groups analyzed; usually the most common finding was chronic inflammation but in that context we did not considered this; to perform other biopsies in the groups analyzed we considered only the PSA velocity/PSA increasing.

Then we considered the repeated biopsies in the same patient: among 111 patients underwent to TP biopsy, 14 (13\%) received a second biopsy; among 108 patients of TR group, 21 (19\%) received a second biopsy, 3 (2.8\%) received a third biopsy. In Table 2 we report the number of cancers detected at first and repeated biopsies in relations to the total number of diagnosed cancers; it shows that overall cancer rate detected at first biopsy, in relation to the total number of diagnosed cancers, is greater in TP group than TR group ( $89.7 \%$ vs $78.4 \% ; \mathrm{p}=0.021$ ); in patients who underwent to further biopsies, overall cancer rate was $10.3 \%$ and $21.6 \%$ in TP and TR group, respectively.

Then we stratified patients by PSA, PSA ratio, DRE/TRUS, LUTS, presence/absence BPH. As reported in Table 3, in patients with "low cancer suspect" (PSA $<10 \mathrm{ng} / \mathrm{ml}, \mathrm{F} / \mathrm{T}>$ $15 \%$, negative TRUS), TP biopsy had more sensitivity to detect cancer than TR biopsy. On the other hand, TR technique detected more cancers in that patients with "high cancer suspect" (PSA > $10 \mathrm{ng} / \mathrm{ml}, \mathrm{F} / \mathrm{T}<15 \%$, TRUS with suspect images). In addition, when DRE was positive, TP biopsy detected more cancers than TR technique ( $55 \%$ vs $43 \%$ respectively). There were no statistically significant differences in BPH between two groups, so, in this report, the $\mathrm{BPH}$ presence did not influence biopsy sensitivity.

Table 3.

Number of cancers/overall number of diagnosed cancers stratified by different parameters.

\begin{tabular}{|l|c|c|c|}
\hline & TP (29) & TR (37) & Risults TP vs TR (\%) \\
\hline PSA $<4$ & $4 / 29(14)$ & $0 / 37(0)$ & 14 vs 0 \\
\hline PSA 4.1-10 & $15 / 29(52)$ & $18 / 37(49)$ & 52 vs 49 \\
\hline PSA $>10$ & $10 / 29(34)$ & $19 / 37(51)$ & 34 vs 51 \\
\hline F/T $>15 \%$ & $13 / 29(44)$ & $3 / 37(1)$ & 44 vs 1 \\
\hline F/T $<15 \%$ & $16 / 29(55)$ & $30 / 37(81)$ & 55 vs 81 \\
\hline DRE negative & $1 / 29(3)$ & $13 / 37(35)$ & 3 vs 35 \\
\hline DRE (BHP) & $8 / 29(27)$ & $7 / 37(19)$ & 27 vs 19 \\
\hline DRE suspected & $16 / 29(55)$ & $16 / 37(43)$ & 55 vs 43 \\
\hline TRUS negative & $2 / 29(7)$ & $1 / 37(3)$ & 7 vs 3 \\
\hline TRUS (BHP) & $2 / 29(7)$ & $4 / 37(11)$ & 7 vs 11 \\
\hline TRUS suspected & $5 / 29(17)$ & $15 / 37(40)$ & 17 vs 40 \\
\hline LUTS (Yes) & $13 / 29(45)$ & $18 / 37(49)$ & 45 vs 49 \\
\hline LUTS (Not) & $16 / 29(55)$ & $19 / 37(51)$ & 55 vs 51 \\
\hline
\end{tabular}

Table 4.

Number of late complications/number of biopsies (\%) in the two groups.

\begin{tabular}{|l|c|c|}
\hline & TP (\%) & TR (\%) \\
\hline Rectal bleeding & $0 / 125(0)$ & $4 / 132(3)$ \\
\hline Dysuria/painful urination & $0 / 125(0)$ & $3 / 132(2.3)$ \\
\hline Acute ritention of urine & $2 / 125(1.6)$ & $3 / 132(2.3)$ \\
\hline Hematuria & $3 / 125(2.4)$ & $3 / 132(2.3)$ \\
\hline $\begin{array}{l}\text { Cardiovascular effects } \\
\text { (hypertensive crisis, extrasystoles) }\end{array}$ & $0 / 125(0)$ & $1 / 132(0.7)$ \\
\hline
\end{tabular}

Then we analyzed complication rate in both groups: early or immediate complication number, directly biopsy related, was low and it interested only TR group. Considering the limited case number, we did not find any statistically significant difference between two groups ( $0 \%$ vs $4.4 \%$; $\mathrm{p}=0.118$ )

As for late complications, TR biopsy was less safe than counterparty transperineal. Overall, in 257 biopsies we detected 20 cases with complications (7.8\%); 5 in TP group whereas 15 in TR group with a statistically significant difference between two men groups ( $p=0.019)$, as shown in Table 4.

In all cases we had minor complications without need for further hospitalization and resolved, however, within the same day of biopsy.

\section{Discussion}

Our retrospective study had the aim to compare sensitivity and early/late complications rates between transrectal (TR) and transperineal (TP) prostate biopsy.

Since the initial description of the systematic biopsy protocol by Hodge et al. (1989), few studies have been conducted on transperineal biopsy. Kojima et al. (4) in their retrospective work observed a greater improvement, in terms of diagnostic sensitivity, of systematic transperineal 12-core biopsy compared to the standard 6-core biopsy, with an increase of cancer detection rate amounted to $5.2 \%$, assuming at the same time that the conventional 6-core scheme would miss approximately $13.8 \%$ of tumors. In a study published in Urology in 2000 (5) they compared transrectal and transperineal sextant biopsies; the study was performed ex vivo on 40 samples of radical prostatectomy already "diagnosed" for cancer who underwent to sextant biopsies in transverse and longitudinal way, simulating, respectively, the transrectal and transperineal approach. There were no statistically significant differences between the two methods, although transperineal protocol had shown more effective in sampling the peripheral zone of gland. Emiliozzi et al. (6) in 2003 published their scientific work, in which they compared the 6-core transrectal and transperineal biopsies. Overall cancer detection rate was 40\% (43/107 patients); TP biopsy detected $38 \%$ of cancers while transrectal method only $32 \%$. It was demonstrated for the first time that 6-core transperineal biopsy was more sensitive than transrectal counterpart in diagnosing prostate cancer and, at the same time, it began to light the inadequacy of 
the standard transrectal sextant protocol. The first real comparison regarding the diagnostic sensitivity and the complications rate between 12-core systematic transperineal and transrectal biopsy, has been developed in a prospective study of 2008 (7) published in Nature. They compared 200 men divided randomly into two groups of 100 subjects each, to underwent to, respectively, 12-core TR and TP prostate biopsy; no statistically significant differences between the two biopsy approaches were detected. However, the transperineal biopsy showed "most appropriate" in detecting cancer in patients with PSA levels included in the "gray zone" (4.1-10 ng/ml), with a significantly higher proportion of positive core in transition gland zone, generally poorly sampled by TR approach. In our work we found no substantial differences between the two biopsy approaches although the TP method had greater sensitivity in detecting cancer in patients with "low cancer suspicion". On the other hand, TR biopsy has been more sensitive in those cases defined as "at risk" for prostate cancer, especially in the case of PSA > $10 \mathrm{ng} / \mathrm{ml}$, PSA ratio $<15 \%$, TRUS findings of lesions suspicious for prostate cancer. We also found that a number of cancers detected by TR approach was diagnosed only after repeated biopsy, while the initial biopsy was negative for malignancy. Especially among all cancers detected by the two biopsy techniques, the TP technique detected 89.7\% of the tumors as early as the first biopsy compared to $78.4 \%$ of cancers diagnosed by TR method when biopsy was performed for the first time. Similarly, only $10.3 \%$ of cancers were detected by TP repeated biopsies compared to $21.6 \%$ of cancers diagnosed when the TR biopsy was repeated. So we could say that the TP method is more sensitive than the TR one, allowing for faster cancer diagnosis and at the same time reducing the need to submit the same subject to additional biopsies before to reach the diagnosis of prostate cancer.

We also assessed the possible presence of BPH among patients of our study; the rate of the disease in our sample was approximately 44\%, absolutely hand in hand with the data reported in the literature. According to our evaluations, the presence of disease has any influence on the sensitivity of biopsies. Some scientific papers, reported some "difficulties" of TR traditional sextant biopsy in detecting cancer in the presence of $\mathrm{BPH}$, as the needle biopsy tended to sample the central zone of the gland, the seat of the elective process of benign proliferation. The transrectal protocol we used, allowed a proper sampling of the peripheral zone, tending to spare the central portion of the prostate, and this was possible by directing the bioptic needle more laterally, as among other things, Stamey suggested in his study in 1995 (8).

When we compared the percentage of positive cores there were no statistically significant differences between the two methods, even if TR biopsy "sampled" a greater number of cancer positive core (16.7\% vs. $23.1 \%$ respectively), and this could be due to the fact that with one hand by TP approach is more difficult to sample the base of the gland, while on the other hand the transition zone is better sampled and it is interested by prostate cancer in a relatively limited number of cases (approximately $24 \%$ of all prostate cancers).
Few studies have evaluated the differences in complications between the two biopsy methods; in a paper of 2005 (9), was evaluated retrospectively the complication rate between sextant TR and TP biopsies. In a total of 197 biopsies (81 transperineal and 116 transrectal), no statistically significant differences were recorded between the two approaches, with an overall rate of complications of $22.2 \%$ and $19.8 \%$ respectively for the TP and TR approach. Considering, however, the relatively small sample of patients, it was concluded that both methods were safe. Most frequent complication TP biopsy related was gross hematuria without any statistically significant differences with TR method. In literature they report a greater risk of serious complications related to TR biopsy, in particular urosepsy and rectal bleeding. Infective complications, today are less frequent thanks to antibiotic prophylaxis and pre-biopsy enema, with a rate of $2.2 \%$ in the case of TR approach, whereas this percentage is significantly lower with TP approach.

In our study, we evaluated early or immediate complications and late complications arisen during post-biopsy observation. We did not find any statistically significant difference in early complications but in TP group there were no complications (0\%) whereas in TR group we found 3 complications (2.2\%), in particular 2 cases of vaso-vagal syncope and 1 patient with intra-glandular bleeding. As regard late complications, we found a statistically significant difference between TP and TR methods (4\% vs $11 \%$, respectively). Hematuria and acute urine retention were most frequent complications in TP group whereas in TR group most frequent complication was rectal bleeding followed by dysuria/strangury, urine acute retention and hematuria. In addition, in TR group we detected two unusual complications: severe headache needing analgesic drugs and one man with cardiac rhythm alterations and hypertensive crisis, quickly resolved after drugs administration. Unfortunately, it was not possible to assess any long-term complications due to the lack of documentation.

As above described, 12-core TP and TR biopsies showed an equivalent sensitivity in overall prostate cancer diagnosis. In general, transrectal biopsy advantage is simplicity of execution and relative needing of anesthesia, whereas main disadvantage is represented by complications rate still relatively high, in particular infections and rectal bleeding. As regards transperineal biopsy advantage is represented by low complications rate whereas main disadvantages are necessity of anesthesia and complexity of execution; however, today refinement of brachytherapy techniques have made it more "family" relationship with this biopsy approach.

\section{Conclusions}

This retrospective comparison between TR and TP biopsy did not show any statistically significant difference in terms of diagnostic sensitivity of prostate cancer. However, TP biopsy detected more prostate cancers at first biopsy than TR biopsy ( $89.7 \%$ vs $78.4 \%$ respectively). Moreover, TP biopsy showed more diagnostic sensitivity in those patients with "low suspect" for prostate cancer than TR biopsy (PSA $<10$ ng/ml, F/T > 15\%, TRUS 
negative for suspected lesions). This latter instead, detected more cancers in those patients with higher suspect for prostate cancer (PSA > $10 \mathrm{ng} / \mathrm{ml}, \mathrm{F} / \mathrm{T}<15 \%$, TRUS with abnormal lesions).

Today, the Urologist has more instruments to study a patient with a suspect prostate cancer, for example RMN multiparametric or fusion biopsy. However, not all centers can enjoy these instruments so we think that is important to optimize diagnosis, mostly in low risk patients who represent the majority of patients who come to our observation.

In conclusion, the Urologist has the final choice in deciding the most appropriate biopsy technique, especially in a context like the present in which more and more patients, not necessarily affected by cancer, underwent prostate biopsy. Therefore, should be desirable the use of a technique that combines an appropriate diagnostic sensitivity to a low complication rate: transperineal biopsy seems to be able to satisfy this "compromise".

\section{REFERENCES}

1. Hodge KK, McNeal JE, Terris MK, Stamey TA. Random systematic versus directed ultrasound-guided transrectal core biopsies of the prostate. J Urol. 1989; 142:71-5.
2. Borboroglu PG, Comer SW, Rffenburgh RH, et al. Extensive repeat transrectal ultrasound guided prostate biopsy in patients with previous benign sextant biopsies. J Urol. 2002; 60:93-7.

3, Durkan GC, Sheikh N, Johnson P, Hildreth AJ, Greene DR. Improving prostate cancer detection with an extended-core transrectal ultrasonography-guided prostate biopsy protocol. BJU Int. 2002; 89:33.

4. Kojima M, Hayakawa T, Saito T, et al. Transperineal 12-core systematic biopsy in the detection of prostate cancer. Int J Urol. 2001; 8:301-7.

5. Vis AN, Boerma MO, Ciatto S, et al. Detection of prostate cancer: a comparative study of the diagnostic efficacy of sextant transrectal versus sextant transperineal biopsy. Urology. 2000; 56:617-21.

6. Emiliozzi P, Corsetti A, Tassi B, et al. Best approach for prostate cancer detection: a prospective study on transperineal versus transrectal six-core prostate biopsy. Urology. 2003; 61:961-6.

7. Takenaka A, Hara $R$, Ishimura $T$, et al A prospective randomized comparison of diagnostic efficacy between transperineal and transrectal 12-core prostate biopsy. Nature. 2008; 11:134-138.

8. Stamey TA. Making the most out of six systematic sextant biopsies. Urology. 1995; 45:2-12.

9. Miller J, Perumalla C, Heap G. Complications of transrectal versus transperineal prostate biopsy. ANZ J Surg. 2005; 75:48-50.

\section{Correspondence}

Carmelo Agostino Di Franco, MD

carmelo_difranco@tiscali.it

Hussein Jallous, MD

Daniele Porru, MD

Giovanni Luca Giliberto, MD

Tiziano Cebrelli, MD

Bruno Rovereto, MD

Urology Department, IRCCS Policlinico S. Matteo, Pavia, Italy

Carmine Tinelli, MD

Biometrics and Medical Statistics Service, IRCCS Policlinico S.Matteo,

Pavia, Italy 